\title{
Cognitive Radio
}

\author{
Kevin Curran, Gary O’Callaghan, Fionnuala McCullough \\ University of Ulster, Northern Ireland, UK \\ Tel: +44 $2871375565 \quad$ E-mail: kj.curran@ulster.ac.uk
}

\begin{abstract}
Cognitive radio is where wireless devices are sufficiently computationally intelligent about radio resources and related computer-to-computer communications to detect user communications needs as a function of use context, and provide radio resources and wireless services most appropriate to those needs. Its roots came from the discovery by regulatory bodies in various countries which found that most of the radio frequency spectrum was inefficiently utilised. For example, cellular network bands are overloaded in most parts of the world, but amateur radio and paging frequencies are not. Independent studies concluded that spectrum utilization depends strongly on time and place. Moreover, fixed spectrum allocation prevents rarely used frequencies from being used by unlicensed users, even when their transmissions would not interfere at all with the assigned service. This was the reason for allowing unlicensed users to utilize licensed bands whenever it would not cause any interference.

Cognitive radio can monitor, observe, and react to events in a specified environment. The fundamental benefits are the ability to self-adapt, self-manage, and self-optimize under normal conditions, and the ability to self-diagnose and self-heal when unusual problems arise e.g. base stations can automatically adjust operating range (e.g., by controlling transmit power, receiver sensitivity, data rate, frequency channel selection) to compensate for neighboring base stations that fail. In contrast to many wireless systems where static frequency channels are pre-selected in advance and tedious frequency planning is mandatory to minimize interference from equipment operating in adjacent coverage areas. CR can achieve a performance that is close to a licensed band even when using an unlicensed band due primarily to its inherent ability to switch to best channels. This paper provides an overview of Cognitive Radio.
\end{abstract}

Keywords: Cognitive radio, Software defined radio, Wireless, Networks

\section{Introduction}

The term cognitive radio (CR) was invented by Joseph Mitola (Mitola et al., 1999). CR refers to a software based radio system which is capable of sensing factors in its environment such as geographical location and the RF characteristics of other radio devices in the same locale, the CR device can then alter its power, frequency, modulation and other parameters to dynamically reuse available radio spectrum (Mannion, 2004). CR builds on the invention of Software Defined Radio (SDR), whereby for example the software in a cell phone defines the parameters of operation when the user moves from place to place. In the US the development CR is led by the Federal Communication Commission (FCC), who deal with commercial applications, and the Defence Advanced Research Projects Agency (DARPA), who deal with military applications (Mannion, 2004).

Cognitive radio can monitor, observe, and react to events in a specified environment. The fundamental benefits are the ability to self-adapt, self-manage, and self-optimize under normal conditions, and the ability to self-diagnose and self-heal when unusual problems arise e.g. base stations can automatically adjust operating range (e.g., by controlling transmit power, receiver sensitivity, data rate, frequency channel selection) to compensate for neighboring base stations that fail. In contrast to many wireless systems where static frequency channels are pre-selected in advance and tedious frequency planning is mandatory to minimize interference from equipment operating in adjacent coverage areas. CR can achieve a performance that is close to a licensed band even when using an unlicensed band due primarily to its inherent ability to switch to best channels (Devroye et al., 2006). This paper will present at overview of cognitive radio but first we introduce the concepts of licensed and unlicensed frequency bands and software defined radio.

\section{Licensed and Unlicensed Frequency Bands}

It can be extremely difficult to locate radio spectrum and even harder to find spectrum that is available worldwide. Spectrum allocation in the international community is controlled by multiple regulatory bodies such as the FCC in U.S., MKK in Japan and CEPT in Europe). The Licensed bands are generally not prone to interference problems but premium licensed bands can be costly. For example, $2.5 \mathrm{GHz}, 3.5 \mathrm{GHz}$ (outside U.S.) and the $4.9 \mathrm{GHz}$ (public safety) are popular licensed bands for wireless access e.g., Wi-Max, wireless DOCSIS. The license-free frequency bands however are 
widely employed and they provide dynamic, opportunistic access to spectrum for a limited time period. There are benefits such as large-scale frequency planning is avoided and ad-hoc networks also become possible. The $5 \mathrm{GHz}$ band is increasingly used to support wireless backhaul transmissions e.g., multihop mesh networks. It is becoming increasingly deregulated and in the UK market forces are starting to have a bigger say in the use and allocation of radio spectrum e.g. the UK recently deregulated $865-868 \mathrm{MHz}$ band for RFID use.

In the US the FCC assigns users to specific frequencies which include AM, FM radio, shortwave, citizens band, VHF and UHF for TV and bands for cellular phones, GPS, air traffic control, security alarms and radio controlled keys. With the development of new wireless devices the demand on the radio spectrum will only continue to increase (Ashley, 2006). Legacy hardware can exacerbate this shortage, for example vacuum tube TV sets in the 1950's forced new transistor based model to receive only VHF signals. Such hardware related inflexibility is now addressed by software based wireless designs. The next generation wireless technology called Software Defined Radio (SDR) uses embedded signal-processing algorithms to sift out weak signals and reconfigurable code structures to receive and transmit new radio protocols (Ashley, 2006). SDR code and other programmable radio frequency front end interface technologies on laptops could receive TV signals. If fitted with an SDR RF card, for instance, a laptop could then be used as a cellular handset or base station. CR advances this situation by allowing the SDR system to reconfigure its analog RF output, and incorporate self awareness, transmission protocols and etiquette. This would free the user a controlling network and their contract fees (Ashley, 2006).

In 2004, the FCC commissioned a Notice of Proposed Rule Making for how CR could be realized. This coincided with other work to open up new radio spectrum to commercial use, e.g. opening spectrum between 3.1 and $10.6 \mathrm{GHz}$ to commercial use for ultra wideband signalling. In their Spectrum Policy Taskforce report of 2002 the FCC recommended a migration towards a "policy based" solution allowing an opportunity to seamlessly move across spectrum using policy engines to check whether this is permissible (Mannion, 2004). The FCC has identified much potential in allowing the $6 \mathrm{MHz}$ wide band in the UHF band currently assigned for television to be opened up for to secondary markets for last mile data access. This would give the $54 \mathrm{Mbps}$ available with WiFi but the bandwidth delivered would be useful especially in rural areas (Mannion, 2004). It is worth noting that the entire radio spectrum up to $100 \mathrm{GHz}$ only $5-10 \%$ is in use at any given instant (Bing, 2004).

Software Defined Radio will allow the industry to find more creative and efficient use of the airwaves, which in turn, will continue to bring benefits to consumers however software must have sufficient controls so that devices cannot be modified to operate outside FCC-approved parameters. In September 2005, Cisco received the first FCC certification for 802.11a SDR.

\section{Cognitive Radio}

Software defined radio (SDR) is seen as a key technology for future wireless communications (Bing, 2004). SDR hardware is largely reconfigurable and reprogrammable by software. This opens the way for new services and prolongs the lifespan of wireless devices. A device with flexible radio hardware is capable of running different applications, much like PC hardware. A high degree of reconfiguration requires support for different system functionalities. Multi-band systems support more than one frequency band and multi-homing systems support more than one radio standard or service. An example of SDR technology is Motorola's Canopy product (Note 1). Cognitive Radio (CR) is a concept with which a network or wireless node changes its transmission parameters without interfering with licensed users. The alteration of these parameters is based on several factors found in the external and internal radio environment. Examples of these factors are the radio frequency spectrum, user behaviour and network state. The main functions that a Cognitive Radio can perform are:

(1) Spectrum Sensing - Detecting unused spectrum and sharing it without interference to others.

(2) Spectrum Management - Capturing the best spectrum to meet user requirements.

(3) Spectrum Mobility - a cognitive radio user exchanges the frequency of the operation.

(4) Spectrum Sharing - Provides a spectrum scheduling method.

Smart radio that leverages on SDR hardware to perform intelligent and opportunistic sharing of unlicensed radio spectrum employs real-time interaction with its environment to determine transmitter parameters such as frequency, power, modulation and learns when to operate and when to interrupt service. Smart radio must rely on intelligent protocols to adapt spectrum use in response to location and operating environment ultimately leads to "plug and play" wireless systems which require minimum or virtually no manual intervention or frequency management during deployment.

Potential military applications for CR are being developed by DARPA in their XG (next generation communications) in order to allow multiple users to share spectrum in a way that coexists and complements sharing protocols used in WiFi technologies (Mannion, 2004; Bing, 2004). CR is capable of reconfiguring its communication functions based on prior experience, so in the case of car radio, CR would allow the device to build up a database of propagation characteristics, signal strength of different transmission bands throughout car journeys. It could then use this information to decide how 
best to transmit at different times of the day and in different places. This dynamic use of bandwidth will free up the radio spectrum and allow for more RF options which are more dependable and considerably cheaper than at present (Ashley, 2006).

In traditional wireless systems most of the intelligence to make the network operate efficiently resides centrally in the network. In CR by contrast this intelligence would reside in the handsets, laptops or wireless devices. A cognitive controlled subsystem controls the SDR allowing a CR unit to detect RF networking opportunities wherever it finds itself. It is envisioned that $\mathrm{CR}$ devices will exist in networks of other CR devices with each contributing to and taking information from a central database which contains the information necessary to transmit effectively in a particular location at a particular time (Ashley, 2006). Although the communications and wireless industries are now only beginning to take notice of cognitive radio, the U.S. government is already interested in the possibilities that CR offers. Cognitive radios are thought to be a powerful tool for explaining and solving general and specific spectrum access issues, an example of this is locating an open frequency and using it. It improves current spectrum use e.g. fills in the unused spectrum and leaves spectrum that is already in use alone and can improve wireless database performance with increased user throughput and system consistency. The FCC in the US is seeking to remove regulatory impediments in order to continue development and deployment of cognitive radios. Cognitive radios are a possible solution to interoperability issues that are present in public safety organisations especially first responders.

\section{Conclusion}

Cognitive radio has the potential to allow multidimensional reuse of spectrum in space, frequency and time and remove the spectrum and bandwidth limitations that slowed broadband wireless development around the world. This new software is closely related to Software- Defined Radio (SDR). SDR is the software found within a cell phone which operates in real time as the user goes from location to location. Cognitive Radio is more intelligent however as it is a radio that is aware of and can sense its environment; learn from its environment, and perform functions that best serve its users. Although Cognitive Radio can be described as software defined radio, there are problems in designing high quality spectrum sensing devices and algorithms for exchanging spectrum sensing data between nodes. Applications of spectrum sensing include emergency networks, Wireless LANs, higher throughput and transmission distance extensions.

Cognitive Radio is poised to take advantage of the increasing deregulation of radio spectrum to provide high-speed broadband services. It can potentially lead to virtually unlimited wireless bandwidth when spectrum is used and reused more efficiently and co-operatively and ultimately increase capacity and efficiency as a direct result of being able to switch between momentarily idle channels in different portions of radio spectrum for short period of usage. This will solve two key problems in multihop networks which are the ability to operate on multiple non-interfering channels removes the bandwidth penalty associated with single-channel multihop systems and the ability to sense for spectrum availability and switch to different channels dynamically provides an excellent solution to wireless Denial of Service (DoS) attacks and network intrusion problems. The technical foundations for Cognitive Radio have been established with the development of Wireless LANs, where dynamic frequency selection and transmit power control are core features. Software functions like filtering, band selection and interference mitigation need to be further developed into this model however many technical hurdles still remain to be overcome such as determining an acceptable level of interference with other radios in the same locale so that it can stay within an acceptable threshold.

\section{References}

Ashley, S. (2006). Cognitive Radio, Scientific American, Vol. 294, No. 3, March 2006.

Bing, B. (2005). All in a Broadband Wireless Access Network, December 2005, ISBN: 0976675218.

Devroye, N., Shyy, D. and Dunyak, J. (2006). Limits on Communications in a Cognitive Radio Channel, IEEE Communications Magazine, Vol. 44, No. 6, pp. 44-49 June 2006.

Mannion, P. (2004). EETimes - Sharing spectrum the smarter way, $4^{\text {th }}$ of May 2004.

http://www.eetimes.com/showArticle.jhtml? article ID=18700443.

Mitola, J. and Maguire, G. (1999). Cognitive Radio: Making Software Radios More Personal, IEEE Personal Communications, Vol. 6, No. 4, 1999.

Tanenbaum, A.S. (2006). Computer Networks, Fifth edition, Prentice Hall.

\section{Notes}

Note 1. http://motorola.canopywireless.com/ 